\title{
Shape of a bistable composite tape-spring in folding
}

\author{
B. Wang*, K. A. Seffen ${ }^{\dagger}$, S. D. Guest ${ }^{*}$ \\ Department of Engineering, University of Cambridge, Cambridge, CB2 1PZ, United Kingdom
}

A composite tape-spring structure is a thin-walled, laminated open slit tube. With fibres oriented at $\pm 45^{\circ}$, it is stable in both the extended and coiled configurations. In this research, we devise a simple 'free' bending system with minimal constraints to evaluate the folding nature of composite tape-springs. The shape of the tape-spring is characterised by considering both the shape during folding and the final folded shape. Experiments are carried out on composite tapes with different geometries: a finite element model is established and calibrated using the experimental results; a parametric study on the folded tape shape is performed based on a theoretical model to evaluate the effects of the initial geometry. Torsional buckling is clearly observed, and complemented with details from the FE model. Here, we show good agreement between experiments, simulation and theoretical analysis.

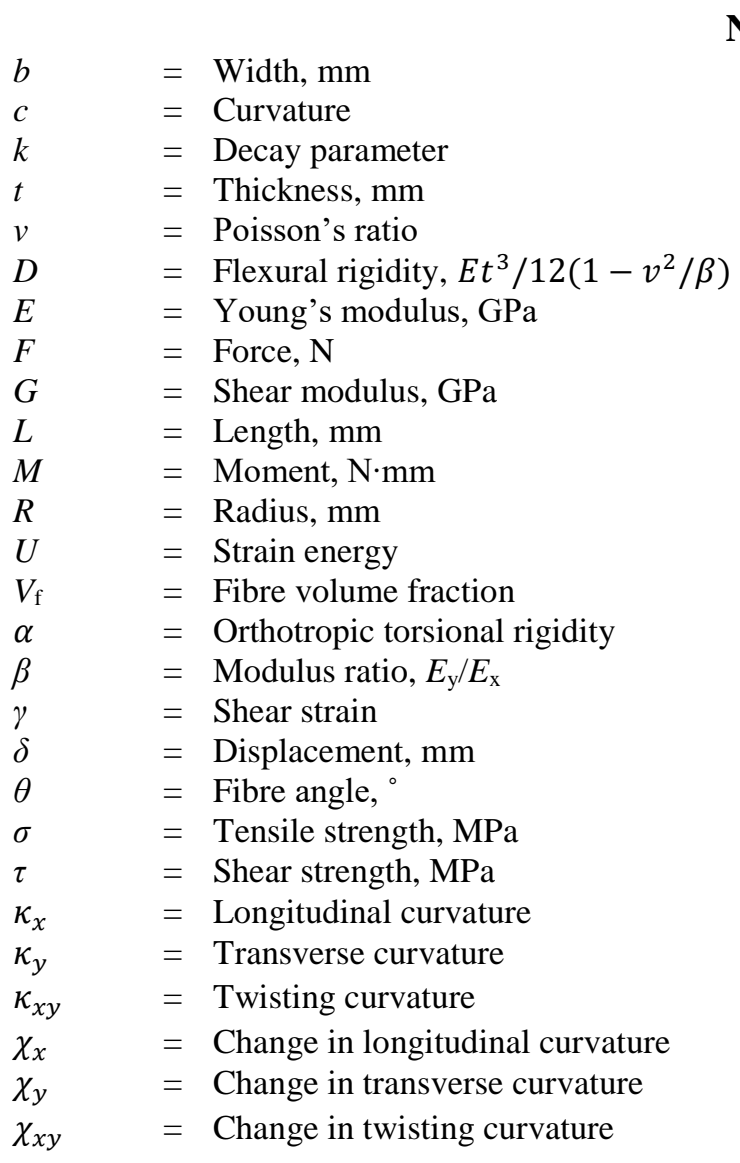

\footnotetext{
* Research Associate, Department of Engineering, University of Cambridge, AIAA Member 905310.

${ }^{\dagger}$ Reader in Structural Mechanics, Department of Engineering, University of Cambridge, AIAA Member 241666.

* Professor in Structural Mechanics, Department of Engineering, University of Cambridge.
} 


\section{Introduction}

$\mathrm{C}^{\mathrm{s}}$ OMPOSITE materials have great potential in aerospace engineering for reducing weight and complexity, and for improving aerodynamic efficiency and shape adaption [1]; morphing composite structures in particular, are intended for extensive use in deployable air- and spacecraft structures. For decades, thin metallic tape-spring structures have been used to construct storable tubular extendible members (STEMs) [2]. More recently, composite tape-springs have been considered for lightweight, low cost and efficient deployable booms [3]. The folding behaviour of a tape-spring structure is highly nonlinear and directionally dependent [4]. For a tape with or without initial longitudinal curvature, bending which generates positive Gaussian curvature is recognised as equal-sense bending, whilst opposite-sense bending induces negative Gaussian curvature.

The elastic folding of an isotropic tape-spring is very well understood. It eventually buckles to form a localised folded region that is approximately uniformly curved longitudinally, with a radius of curvature almost equal to the transverse radius of the undeformed cross-section [5]. The analytical moment-rotation relationships for symmetrical tape bending have been developed by Rimrott [6], Calladine [7], Mansfield [8] and more recently by Seffen [9]. Unsymmetrical bending characterised by tape twisting has been studied also by Mansfield in consequence to uniform equal-sense bending and buckling in a torsional mode.

In this research, we characterise the folding of bistable composite tape-spring structures, which are envisaged as replacements for side lock-stays or link-stays within large aircraft landing gear [10]. Unlike normal three-point bending tests, we established a "free" three-point bending set-up with minimal end constraints, in order to permit twisting throughout: this is not the same as Mansfield's uniform curving but it represents a step that way.

Initial findings have been recently reported [11,12]; here, we provide further details including how the tape shape changes during folding, and characterisations of the final folded static shapes. Firstly, the mechanical properties of the composite layup are evaluated with embedded strain gauges, and these data are used to establish the finite element (FE) model. The "free" bending tests are carried out on a range of composite tapes and complemented by an FE model, following the modified Naik's approach [13] and calibrated by experimental results. The folded tape shape is evaluated both experimentally and numerically. A parametric study is performed to understand how the folded shape changes with initial geometry: subtended angle, radius and thickness.

\section{Theoretical}

Fig. 1 schematically presents a typical force-displacement profile from "free" three-point, equal-sense bending of a tape-spring structure, made from either isotropic or orthotropic materials [12]. The tape ends are free to rotate and to approach one another, but are restrained linearly against the centrally applied, vertical force, $F$. Four stages of deformation are experimentally observed: stage $\mathrm{A}$ is initial, symmetrical bending with smooth and uniform deformation along the tape; stage B commences when torsional buckling initiates, and twisting deformation gradually follows. Twisting then diminishes during stage $\mathrm{C}$ as the fold begins to localise and become well formed as the applied force drops. A minimum force is then expressed at the onset of stage D where the curved fold is complete and central, and separated by transition, or "ploy", regions, Fig. 2, on either side: "point-like" folding then proceeds under increasing force.

We are first interested in the linear limit of the applied force, defined in the first instance by the force at buckling $F_{\text {crit. }}$ Using the approach outlined in [12], we express first the total strain energy per unit length of tape as $U$ :

$$
\begin{aligned}
& U=\frac{D b}{2}\left[\kappa_{x}^{2}+2 \alpha \kappa_{x y}^{2}+\phi\left(\kappa_{x y}^{2}-\frac{\kappa_{x}}{R}\right)^{2}\right] \\
& \text { with } \phi=b^{4}\left(1-v^{2} / \beta\right) /\left(60 t^{2}\right)
\end{aligned}
$$

This assumes instantaneously uniform curvatures, $\kappa$, at the centre of the tape-spring, which informs the bending moments and ultimately the applied force. If no axial torque is applied, we must observe a minimum in $U$ with respect to twisting curvature, $\kappa_{x y}$, [8]. If no axial torque is applied,

$$
\frac{\partial U}{\partial \kappa_{x y}}=\kappa_{x y}\left[\alpha+\phi\left(\kappa_{x y}^{2}-\kappa_{x} / R\right)\right]=0
$$

Two solutions are obtained, either (i) $\kappa_{x y}=0$, corresponds to initial bending in stage A; or (ii) $\kappa_{x y}^{2}-\kappa_{x} / R=$ $-\alpha / \phi$, represents non-zero twisting and a fixed level of Gaussian curvature: this gives the onset of stage B but without capturing the localisation further into the deformation mode. 


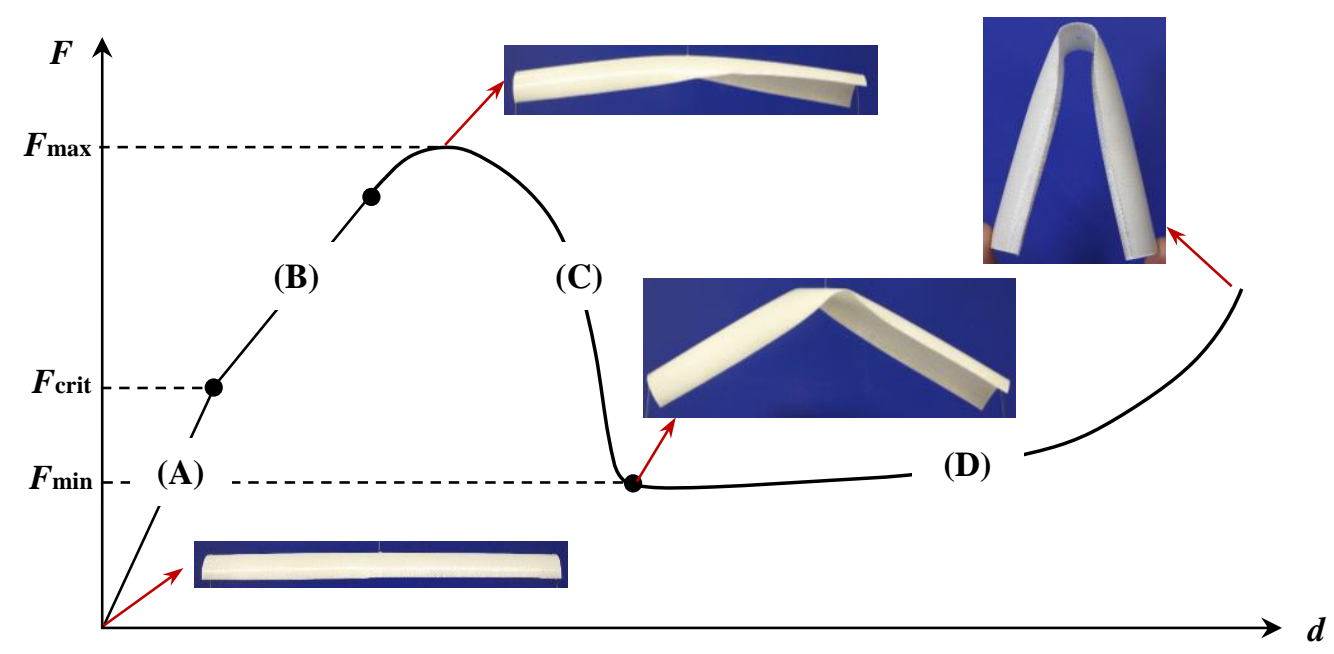

Fig. 1 Schematic representation of force-displacement profile during equal-sense bending of a tape-spring structure according to the set-up in Fig. 3. Insets show examples of deformed shapes at different stages of a woven composite tape-spring with fibres in $\pm 45^{\circ}$.

The applied moment for each solution found by substituting $\kappa_{x y}$ and then differentiating $U$ with respect to $\kappa_{x}$ :

$$
M_{\mathrm{A}}=D b \kappa_{x}\left[1+\frac{\phi}{R^{2}}\right]
$$

and

$$
M_{\mathrm{B}}=D b\left[\kappa_{x}+\alpha / R\right]
$$

They are linear and differ in gradient. The rest of stages B and C defy straightforward modeling because of how twisting and bending interact and localise; the untwisted fold of stage D, however, allows the applied force to be estimated from the leverage of the fold behaving as a point-like hinge with an elastic torsional resistance equal to the well-known transverse bending moment [12].

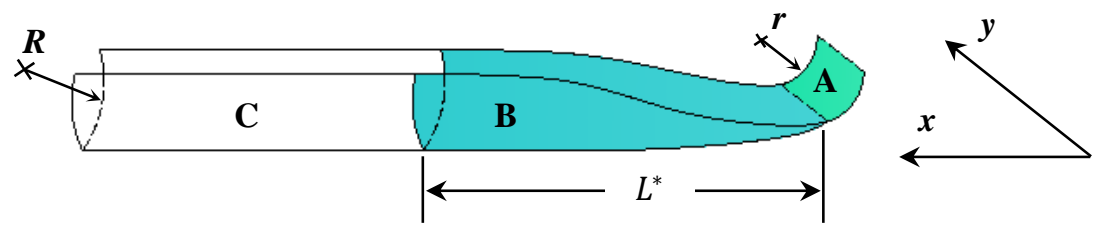

Fig. 2 Schematic folded tape-spring shape: the shaded region $B$ is the ploy region up to the start of the circular fold region $A$ with radius $r$, and region $C$ is the natural end with radius $R$. The length of the ploy region is $L^{*}$ where the transverse curvature ranges from 0 to $1 / R$.

The folded tape has three clear regions, which are shown for half of a tape in Fig. 2: the fold itself, region A, which connects to the start of the ploy region $\mathrm{B}$ over which the change in transverse curvature decays before the undeformed straight end region $\mathrm{C}$. At the connection between $\mathrm{A}$ and $\mathrm{B} \kappa_{x}=1 / r$ and $\kappa_{y}=0$, which define the boundary conditions for the ploy region. One solution from [12] for this shape is given by:

$$
\begin{gathered}
\chi_{x}=-\frac{k^{2}}{R} e^{-k \frac{\pi x}{b}}\left[1-\cos \left(\frac{\pi y}{b}\right)\right] \\
\chi_{y}=-\frac{1}{R} e^{-k \frac{\pi x}{b}} \cos \left(\frac{\pi y}{b}\right) \\
\chi_{x y}=\frac{k}{R} e^{-k \frac{\pi x}{b}} \sin \left(\frac{\pi y}{b}\right)
\end{gathered}
$$

where, $k$ is a decay parameter given by: 


$$
k^{4}=\frac{B}{36 S} \frac{\beta}{1-v^{2} / \beta} \frac{t^{2} R^{2}}{b^{4}}
$$

which allows the ploy region length, $L^{*}$, in Fig. 2 , to be estimated as:

$$
L^{*}=\frac{b^{2}}{\pi}\left[\frac{1-v^{2} / \beta}{120 \beta}\right]^{1 / 4} \sqrt{\frac{c}{t}}
$$

\section{Experimental}

\section{A. Production of composite tape-springs}

Our composite tapes use glass fibre (GF) reinforced polypropylene (PP). The layup consists of three layers of plain-weave GF and PP sheet, and Table 1 gives the suppliers materials properties. To produce a tape sample, the layup was placed between two pieces of PTFE coated glass fabric paper, and gradually wrapped and tightened on a cylindrical mould (diameter $25 \mathrm{~mm}$, length $300 \mathrm{~mm}$ ) using heat shrink tape. A jubilee clip was then used to lock the whole layup before curing in a pre-heated fan-assisted oven at $225^{\circ} \mathrm{C}$ for 4 hours. After the mould was released, each sample was manually cut for testing. The composite has a typical fibre volume fraction, $V_{\mathrm{f}}$, of $30 \%$.

Table 1 Materials properties of glass fabric and polypropylene sheet.

\begin{tabular}{lcc}
\hline Materials & GF plain-weave woven [14] & PP sheet [15] \\
\hline Density & $200 \mathrm{~g} / \mathrm{m}^{2}$ & $0.9 \mathrm{~g} / \mathrm{cm}^{3}$ \\
Thickness (mm) & 0.20 & 0.5 \\
Construction warp $\times$ weft $(\mathrm{th} / \mathrm{cm})$ & $7.4 \times 7.4$ & -- \\
Poisson's ratio & 0.2 & 0.42 \\
Elastic modulus $(\mathrm{GPa})$ & 72.4 & 1.53 \\
Shear modulus $(\mathrm{GPa})$ & 30 & 0.54 \\
Thermal expansion coefficient & 5.0 & 84.8 \\
$\left(\mathrm{TEC} \mu \mathrm{m} / \mathrm{m}^{\circ} \mathrm{C}\right)$ & & \\
\hline
\end{tabular}

\section{B. Mechanical properties}

To evaluate the mechanical properties of composite, flat samples were produced with embedded strain gauges. Production of flat samples are similar to Section III.A, using flat steel plate of size $(320 \times 50 \times 3 \mathrm{~mm})$ as a mould, and strain gauges were embedded in the layup. This arrangement ensured direct contact between the strain gauges and samples to minimise effects from using adhesives, as well as keeping minimal effects to the composite properties. The embedded strain gauge was soldered to a lead pad, and connected to a National Instrument PXIe-1071 series transducer; data were monitored and recorded at $2 \mathrm{~Hz}$. A sample dimension of $250 \times 30 \times 0.65 \mathrm{~mm}$ was employed with a testing span of $190 \mathrm{~mm}$. A $6 \mathrm{~mm}$ strain gauge with resistance $120 \Omega$ was supplied by Tokyo Sokki Kenkyujo Co. Ltd., with a thermal expansion coefficient of $11 \mu \mathrm{m} /\left(\mathrm{m} \cdot{ }^{\circ} \mathrm{C}\right)$, and transverse sensitivity $(K t)$ of $0.1 \%$.

Mechanical tests were carried out using an Instron 4483 universal tensile machine with a $30 \mathrm{kN}$ load cell at room temperature. A constant crosshead speed of $2 \mathrm{~mm} / \mathrm{min}$ was adopted for all tests. Elastic properties in fibre direction were determined by testing samples with warp fibres in the longitudinal and transverse directions following ASTM D3039, the layups being designated as $[0 \mathrm{~F}]_{3}$ and $[90 \mathrm{~F}]_{3}$ separately. Shear properties were derived from tests of inplane shear responses according to ASTM D3518 with warp fibres oriented in $45^{\circ}$, and the layup was denoted as $[45 \mathrm{~F}]_{3}$. Three samples for each test were conducted to evaluate repeatability.

\section{Optical microscopy}

Although plain-weave GF fabric has the same warp and weft yarn count as in Table 1, the mechanical properties of the woven composite are also dependent on the yarn bundle geometry within the cross-section [13], which is evaluated using optical microscopy: flat woven composite samples were embedded into an epoxy matrix, cured and then progressively grounded and polished for examination.

\section{Three-point bending}

The three-point, equal-sense bending set-up is shown schematically in Fig. 3. Long steel wires were attached to each of the three connection points within an Instron Serious 5564 materials testing machine. The speed of displacement was set at $60 \mathrm{~mm} / \mathrm{min}$ and the applied force was recorded using a $100 \mathrm{~N}$ load cell. Each test was repeated three times to evaluate repeatability. 


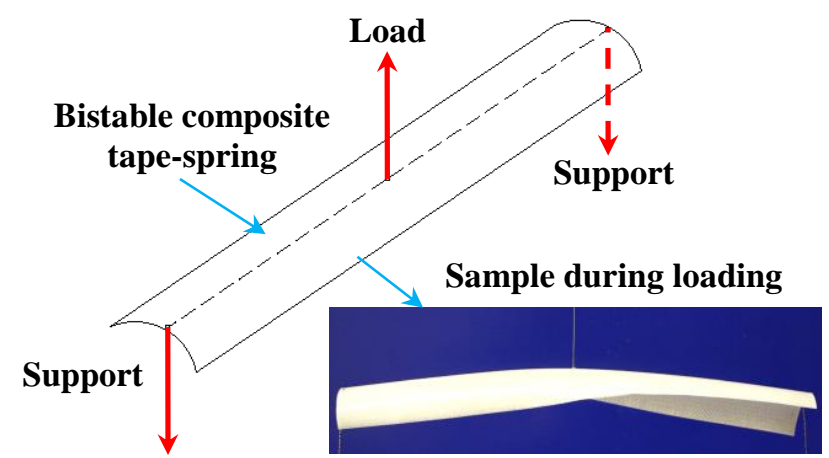

Fig. 3 Experimental setup for the folding of a tape-spring structure under equal-sense bending.

\section{E. 3D laser-scanning}

The folded tape shape of a CTS was recorded using a zSnapper ${ }^{\circledR}$ vario 3D laser-scanner [16]. Fig. 4 schematically shows the experimental setup. The laser projector emits stripes of laser light onto an object, which are deformed by its shape and captured by the camera. Calibration was made by measuring a calibration gauge (with reference points) in different positions using SnapCal software. The calibration data were imported into SingleZsn software, where all sample scans were recorded and constructed into a discrete point cloud [16], and reconstructed using MATLAB [17]. All scans had a resolution of $640 \times 480$ pixels and scanned using without reference mode.

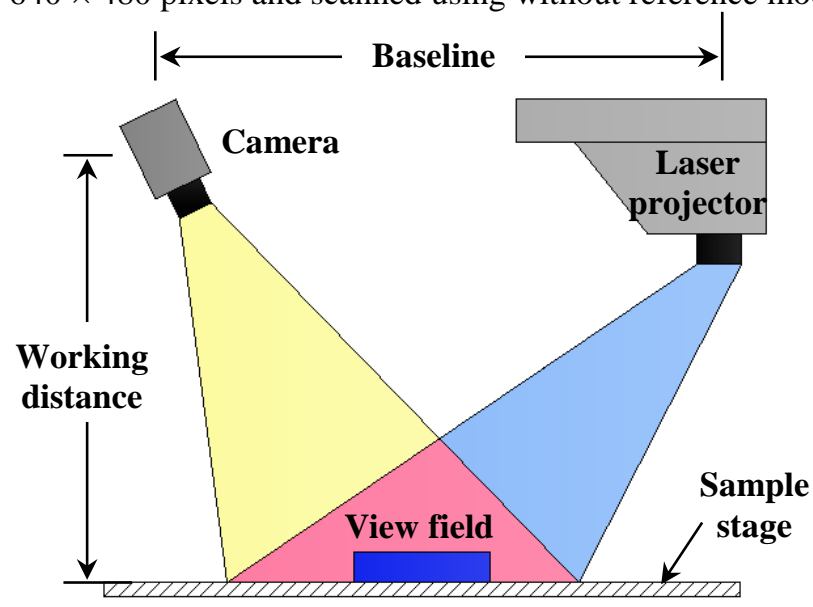

Fig. 4 Schematic representation of 3D laser-scanning setup.

\section{Finite element method}

\section{A. Micromechanics of woven composite}

There have been many attempts to model woven composites, as documented by Ansar et al [18]. Performances of woven composite for specific purposes have been simulated through idealization or homogenisation of geometric parameters and weave architecture at a micro-structural level; while it is complicated by discretising the unit cell into elements and slices, which involves substantial computation at a macroscopic level. Here, woven fabric layers are usually simplified by using multiple unidirectional (UD) fibre layers, which provide close approximation of the membrane stiffness [19,20]. Our FE model is based on the simplified Naik's model [13]: a woven composite ply is composed of one isotropic matrix layer and two UD laminates, which gives effective and reasonable correlation to the real woven composites [20].

Fig. 5 shows the coordinate system and fibre directions. Properties of the optimised UD laminate are determined by mechanical tests and by the micro-mechanical model. Since the well-known rule-of-mixtures is unrealistic in the prediction of transverse modulus for the iso-stress assumption [21], another widely accepted model based on HashinShtrikman (H-S) bounds [22] (also known as the composite cylinder assemblage model) provides an alternative basis. 


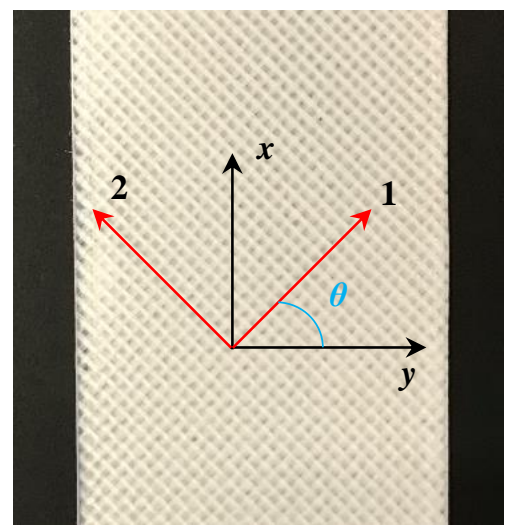

Fig. 5 Coordinate system and fibre directions of the GF/PP woven composite: ' 1 ' denotes the fibre in warp direction; ' 2 ' is the fibre in weft direction; ' $x$ ' is along the sample length and ' $y$ ' is transverse; ' $\theta$ ' defines the fibre angle with $+\theta$ in warp and $-\theta$ in weft.

\section{B. Finite element model}

The FE model is constructed using ABAQUS/Explicit [23]. As discussed above, the woven composite layup $[ \pm 45 \mathrm{~F}]_{3}$ is simplified into UD laminates described as $\left[\mathrm{m} /(45 /-45)_{3} / \mathrm{m}\right]$, where the $m$-ply is the pure PP matrix layer. The actual thickness of each ply is determined from optical microscopy, setting the thickness of the optimised UD layer to be $0.1 \mathrm{~mm}$ and the matrix layer as $0.025 \mathrm{~mm}$.

The model uses S4R reduced integration, four-noded shell elements coupled to hourglass control, finite membrane strains and a geometrically nonlinear analysis. The "stabilise" function minimises instabilities and we specify a damping factor of $1 \times 10^{-6}$. Convergence studies on the mesh size have been carried out to determine acceptable accuracy of the model: an element edge size of $2 \mathrm{~mm}$ and around 2000 elements in total were found to be successful.

The constrained translational degrees of freedom are kept minimal: displacement restrictions in the $z$-direction were applied on two nodes at the centre of cross-section at both ends; one node was able to move towards the other along the $x$-direction. Displacement restriction was also applied to the central node in the $y$-direction. No rotational degrees were constrained. Folding was then achieved by displacing the mid-node of the shell in $z$-direction.

\section{Results and discussion}

\section{A. Mechanical properties of woven composite}

Mechanical properties of the GF/PP composite are evaluated with embedded strain gauges, see Table 2. Though the woven composite samples were fabricated with the same fibre counts in both directions, the Young's modulus in the warp direction is found to be slightly lower than the weft direction: the relative difference in modulus values is 0.81. A similar variance is obtained for the value of Poisson's ratio, with $v_{12}=0.11$ compared to $v_{21}=0.17$. This may be caused by the differences of fibre tow geometry in the warp and weft directions [13], and this is further addressed below. The H-S bounds based micromechanical model (Section IV.A) gives $E_{1}^{W C}=E_{2}^{W C}=13.70 \mathrm{GPa}, G_{12}^{W C}=$ $978 \mathrm{MPa}$, and $v_{12}=v_{21}=0.10$. Despite the experimental system error, these agree well with the mean measured modulus value of $14.53 \mathrm{GPa}$, shear modulus of $824 \mathrm{MPa}$ and Poisson's ratio of 0.14 .

The tensile strength of the composite layup in warp $\left(\sigma_{1}^{W C}\right)$ and weft $\left(\sigma_{2}^{W C}\right)$ directions are $215 \mathrm{MPa}$ and $198 \mathrm{MPa}$, respectively, corresponding to failure strains of $\varepsilon_{1 f}^{W C}=3.5 \%$, and $\varepsilon_{1 f}^{W C}=2.3 \%$, with a shear strength $\left(\tau_{12}^{W C}\right)$ and strain $\left(\gamma_{12 f}^{W C}\right)$ of $42.3 \mathrm{MPa}$ and $26.9 \%$, respectively.

Fig. 6 shows the cross-section of GF/PP composite in both the warp and weft directions. Though the count of filaments is the same in both directions, the tow geometry is clearly different. Table 3 presents the tow geometry of a single woven ply as measured by using FIJI [24]. The fibre tow in warp direction are two times wider and $~ 70 \%$ less in height compared to weft fibre tow. The thickness of a single woven lamina is $0.205 \mathrm{~mm}$, which correlates to the lamina geometry in the FE model.

To clearly define the differences in tow geometry, a unit cell model of the woven composite is established using TexGen [25] base on the measured data in Table 3, and is shown in Fig. 6-c. For the same fibre counts, Table 1, the cross-section area of a single warp fibre tow is $20 \%$ lower than a weft tow, in accordance with the difference in measured modulus values: a more effective and collective response of fibres can be expected in the weft direction under loading, which would result in a higher value of modulus, see Table 2 . The tensile strength in weft direction is 
also lower than warp direction as identified in Table 2. Thus, the discrepancy in modulus, strength and Poisson's ratio values are due to variations in the fibre tow geometry.

Table 2 Mechanical properties of GF/PP composite samples with embedded strain gauges. SE is the "standard error".

\begin{tabular}{ccccc}
\hline Sample No & Strength $(\mathrm{MPa})$ & Strain at failure $(\%)$ & Modulus $(\mathrm{GPa})$ & Poisson's ratio \\
\hline & & & & \\
{$[0 \mathrm{~F}]_{3}$} & $\sigma_{1}^{W C}$ & $\varepsilon_{1 f}^{W C}$ & $E_{1}^{W C}$ & $v_{12}$ \\
1 & 206.68 & 3.55 & 12.79 & 0.11 \\
2 & 206.52 & 3.56 & 12.27 & 0.12 \\
3 & 233.08 & 3.26 & 13.90 & 0.11 \\
Mean $\pm \mathrm{SE}$ & $215.43 \pm 8.83$ & $3.46 \pm 0.10$ & $12.99 \pm 0.48$ & $0.11 \pm 0.01$ \\
& & & & \\
{$[90 \mathrm{~F}]_{3}$} & $\sigma_{2}^{W C}$ & $\varepsilon_{2 f}^{W C}$ & $E_{2}^{W C}$ & $v_{21}$ \\
1 & 196.29 & 2.33 & 15.52 & 0.13 \\
2 & 203.05 & 2.34 & 18.69 & 0.16 \\
3 & 194.8695 & 2.34 & 13.98 & 0.21 \\
Mean $\pm \mathrm{SE}$ & $198.07 \pm 2.52$ & $2.34 \pm 0.01$ & $16.06 \pm 1.39$ & $0.17 \pm 0.03$ \\
& & & & \\
{$[45 \mathrm{~F}]_{3}$} & $\tau_{12}^{W C}$ & $\gamma_{12 f}^{W C}$ & $G_{12}^{W C}$ & -- \\
2 & 41.06 & 27.99 & 713 & -- \\
3 & 43.09 & 25.58 & 847 & -- \\
Mean $\pm \mathrm{SE}$ & $42.30 \pm 0.63$ & $26.92 \pm 0.71$ & $824 \pm 59$ & - \\
\hline
\end{tabular}
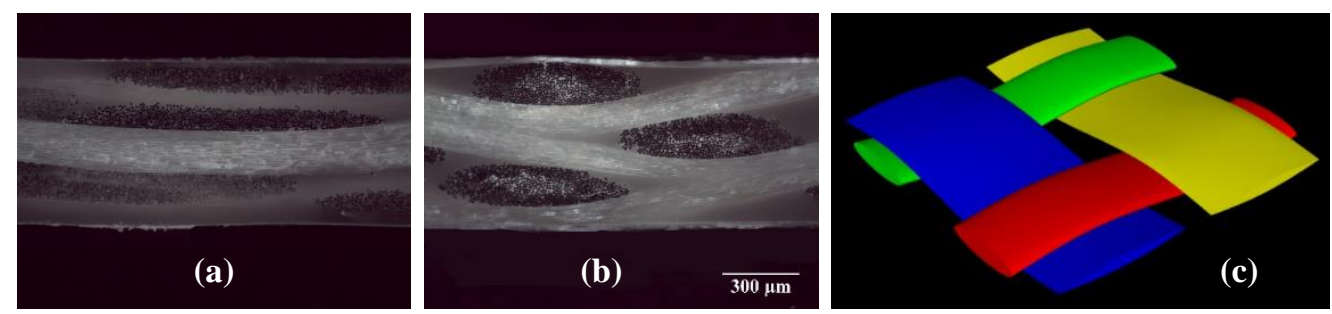

Fig. 6 Cross-sectional of GF/PP woven composite in (a) warp and (b) weft directions, (c) shows the unit cell model constructed base on the data in Table 3.

Table 3 Tow geometry of the plain-weave GF in warp and weft directions.

\begin{tabular}{cccc}
\hline Direction & Tow width, $a_{\mathrm{t}},(\mathrm{mm})$ & Tow height, $h_{\mathrm{t}},(\mathrm{mm})$ & Tow spacing $(\mathrm{mm})$ \\
\hline Warp & 1.285 & 0.092 & 1.332 \\
Weft & 0.669 & 0.153 & 1.332 \\
\hline
\end{tabular}

\section{B. Validation of the FE model}

The folding behavior of composite tapes with lengths of 50,100 and $200 \mathrm{~mm}$ are shown in Fig. 7. The sharp peaks at onset of loading observed in FE model are derived from torsional buckling [11,12]; while Experiments show a gradual and smooth torsional buckling response owing to the presence of imperfections. Apart from the torsional coupling peak, the load magnitudes mostly match each other. Table 4 shows the data points at peaks $\left(F_{\max }\right)$, and valleys $\left(F_{\min }\right)$ extracted from Fig. 7 . It is clear that the model is capable of capturing the main features of composite tape-spring in folding. 


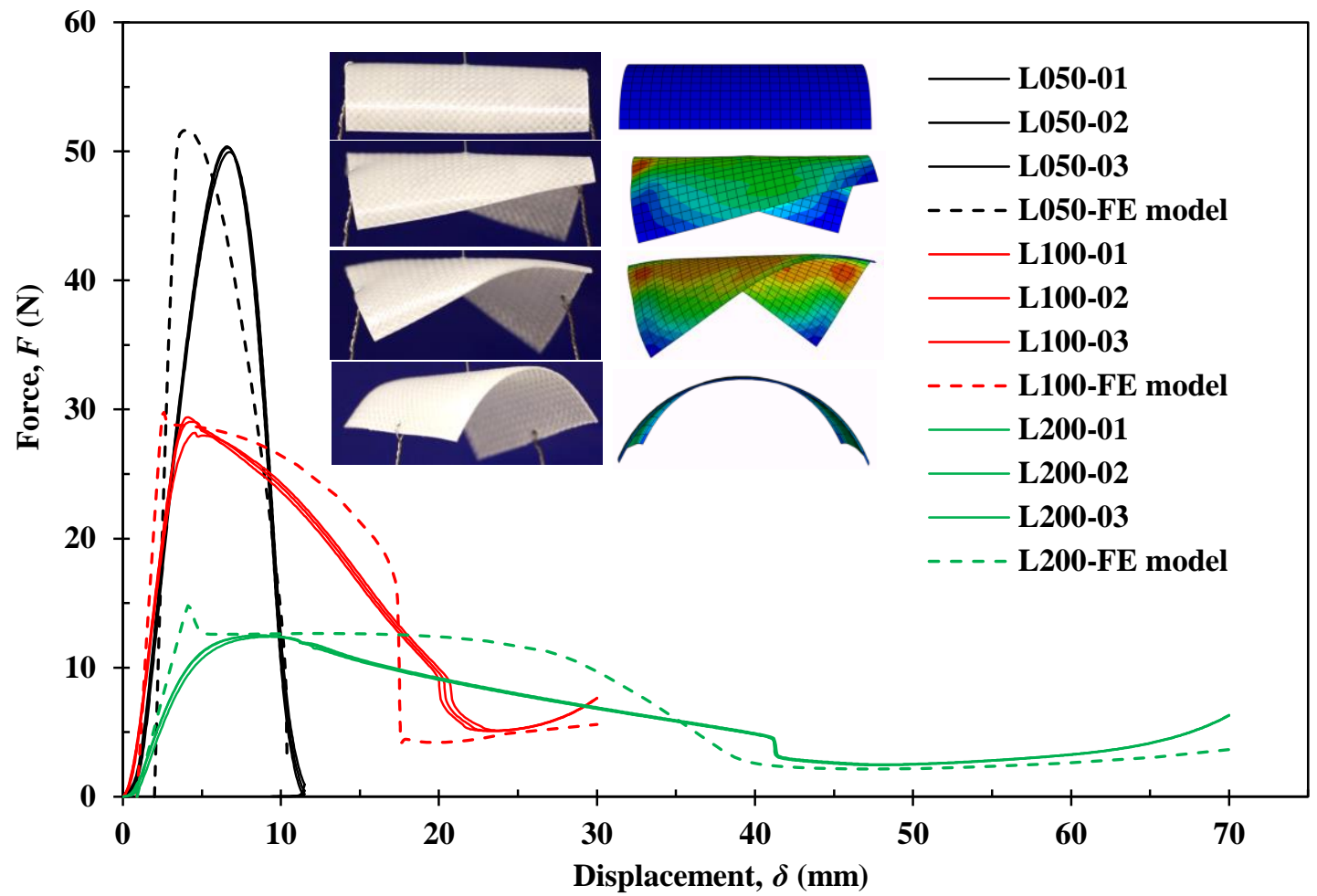

Fig. 7 Force-displacement curves of composite tapes with different lengths from FE model and experiments: inset figures compare shapes of a tape $50 \mathrm{~mm}$ in length.

Table 4 FE data on peaks and valleys during folding of composite tape-springs with different length, compared to expeirments in Fig. 7. SE is the "standard error".

\begin{tabular}{|c|c|c|c|c|c|}
\hline \multirow{2}{*}{$\begin{array}{l}\text { Subtended } \\
\text { angle }\left({ }^{\circ}\right)\end{array}$} & \multirow{2}{*}{$\begin{array}{l}\text { Length } \\
(\mathrm{mm})\end{array}$} & \multicolumn{2}{|c|}{ Peak } & \multicolumn{2}{|c|}{ Valley } \\
\hline & & $F_{\max }(\mathrm{N})$ & $\delta(\mathrm{mm})$ & $F_{\min }(\mathrm{N})$ & $\delta(\mathrm{mm})$ \\
\hline \multirow[t]{15}{*}{180} & 50 & 49.79 & 6.71 & -- & -- \\
\hline & & 50.17 & 6.59 & -- & -- \\
\hline & & 50.22 & 6.60 & -- & -- \\
\hline & Mean \pm SE & $50.01 \pm 0.14$ & $6.64 \pm 0.04$ & -- & -- \\
\hline & $\mathrm{FE}$ & 51.67 & 4.10 & -- & -- \\
\hline & 100 & 28.20 & 4.51 & 5.13 & 23.81 \\
\hline & & 29.05 & 4.40 & 5.09 & 23.46 \\
\hline & & 29.40 & 4.07 & 5.09 & 23.40 \\
\hline & Mean \pm SE & $28.88 \pm 0.36$ & $4.33 \pm 0.13$ & $5.10 \pm 0.01$ & $23.55 \pm 0.13$ \\
\hline & FE & 29.77 & 2.59 & 4.17 & 17.64 \\
\hline & 200 & 12.39 & 9.31 & 3.10 & 41.62 \\
\hline & & 12.48 & 8.97 & 3.24 & 41.42 \\
\hline & & 12.47 & 8.57 & 3.16 & 41.52 \\
\hline & Mean \pm SE & $12.45 \pm 0.03$ & $8.95 \pm 0.21$ & $3.17 \pm 0.04$ & $41.52 \pm 0.06$ \\
\hline & FE & 14.78 & 4.11 & 2.43 & 40.75 \\
\hline
\end{tabular}

\section{Tape shape in folding}

Further insights can be gleaned from plotting the changes in curvature at the central loading point, see Fig. 8. Pure bending in stage A corresponds to curvature changes in both $x$ - and $y$-directions, and the torsional coupling peak is 
clearly demonstrated by the sharp peak in $\chi_{y}$ at displacement of $\sim 3 \mathrm{~mm}$. The tape twists in stage $\mathrm{B}$, reaching a maximum value at $\sim 30 \mathrm{~mm}$ in stage $\mathrm{C}$, before decreasing. In stage $\mathrm{D}$, a minimum force is observed when $\chi_{y}$ approaches $1 / R$ and twisting disappears.

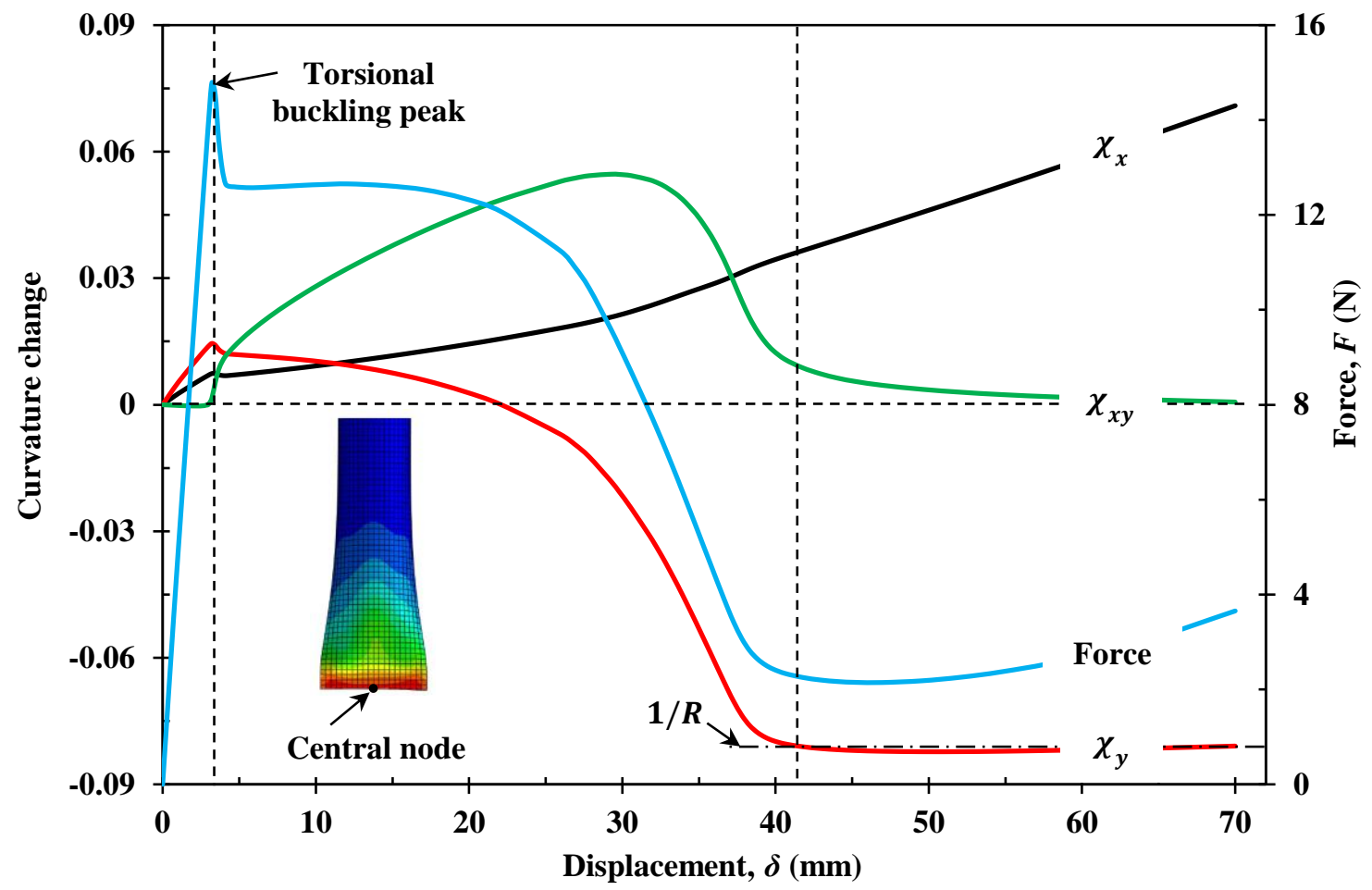

Fig. 8 Curvature changes of the central node in longitudinal $\left(\chi_{x}\right)$, transverse $\left(\chi_{y}\right)$ and twisting $\left(\chi_{x y}\right)$ directions during folding process. Inset shows the position of the central node.

\section{Folded tape shape}

Fig. 9 shows the curvature changes on nodes along central line of a folded tape shape with a folded angle of $\sim 90^{\circ}$. Theoretically, along the central line of a folded tape within region $\mathrm{B}, \chi_{x}=\chi_{x y}=0$, accompanied by an exponential decay in $\chi_{y}$ as defined by $k$. The value of $k$ can be determined by fitting Eq. 6 to the data of curvature changes from the FE model. Fig. 9 shows that there is a transition point T, defined as the maximum derivative value point, which indicates the onset of exponential decay in $\chi_{y}$, and is well-fitted by Eq. 6 with the decay parameter $k=0.62$. In this region, $\chi_{x}$ and $\chi_{x y}$ are also close to zero, which correlates with our assumptions for Eqs. 5 and 7.

The fitting-curve is extended to $\chi_{y}=1 / R$, i.e. point $\mathrm{N}$ where $\chi_{y}=1 / R$, as indicated by the dashed line in Fig. 9 , and defines the theoretical boundary. Thus, there is a transition region $\mathrm{A}^{\prime}$ between regions $\mathrm{A}$ and $\mathrm{B}$. Any differences between the theoretical analysis and real shape is considered to be minor compared to the complexity it would bring mathematically. The ploy length, $L^{*}$, is therefore characterised as the distance between points $\mathrm{N}$ and $\mathrm{P}$. Considering $\chi_{y}<5 \%$ of $\kappa_{y 0}$ as a boundary to the natural end region C, the ploy length $L^{*}=L_{P}-L_{N}$ is found to be $57 \mathrm{~mm}$. This correlates well with the prediction from Eq. 9 which gives the value of $53.7 \mathrm{~mm}$. A parametric study is performed later in Section E. 


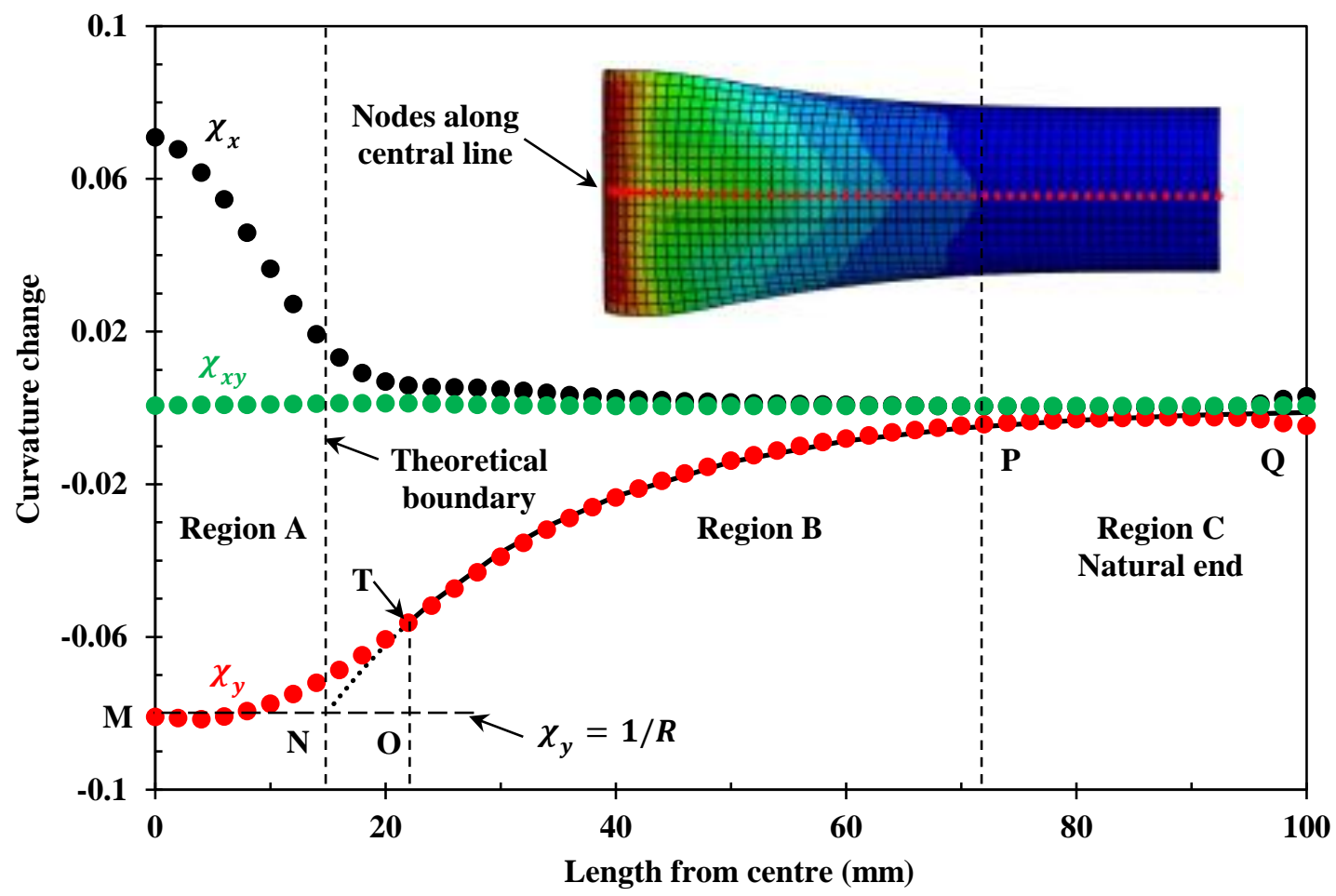

Fig. 9 Curvature changes on nodes along central line of a folded tape. Solid curve is fitted by Eq. 6, dashed line shows an extension of exponential decay.

(a)

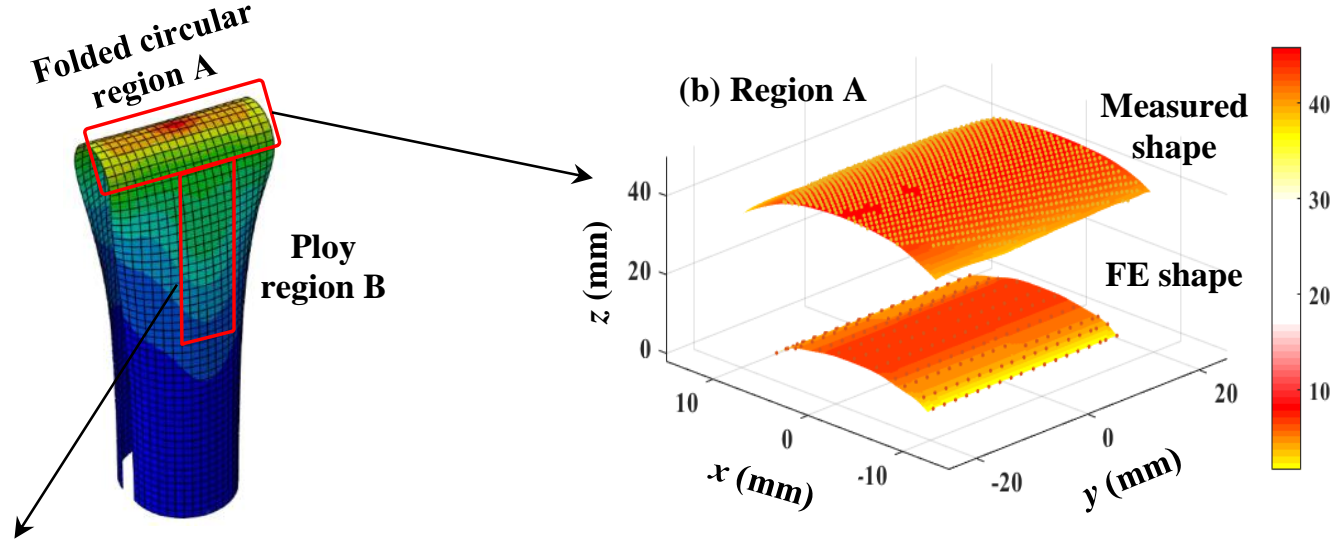

(c) Region B

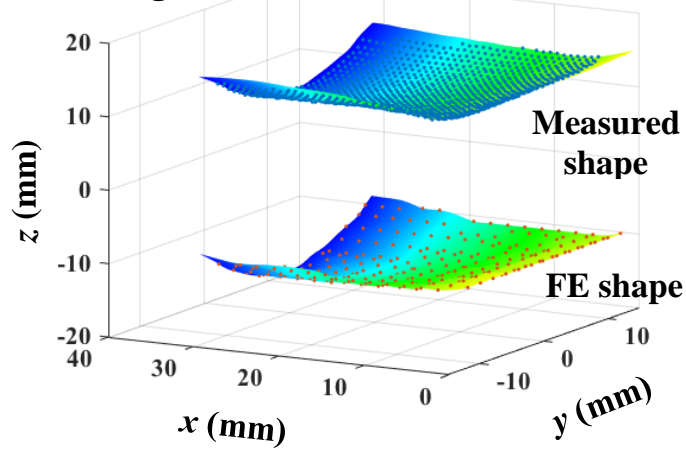

(d)

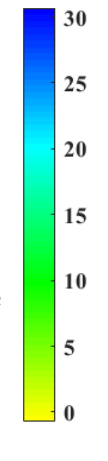

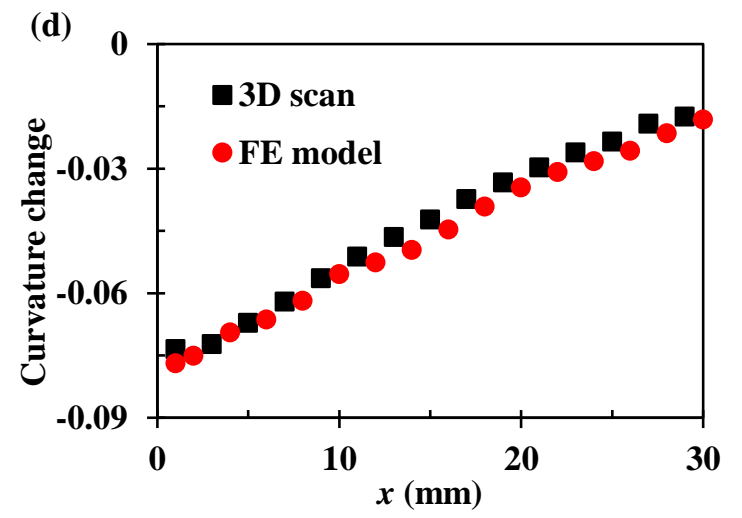

Fig. 10 Folded tape shape: shape of folded CTS of 200 length and subtended angle $180^{\circ}$ from the 3D laserscanning and $\mathrm{FE}$ analysis: points are from scanning. 
The same tape is folded to a larger angle, $180^{\circ}$, and the folded shape is evaluated by using the 3D laser-scanning and the FE model, Fig. 10. Scanned regions of interest lie in the ploy region A and B as highlighted in Fig. 10-a. The shapes of the real sample are then compared to the FE model. In the folded circular central region, Fig. 10-b, the measured shape gives nearly uniform transverse curvature, $\chi_{y}=0.00036$, with $\chi_{x}=0.0452$. The FE shape also indicates similar transverse curvature, $\chi_{y}=0.00039$, while $\chi_{x}=0.0722$. This can be attributed to the differences in the applied boundary conditions, since the central fold region is quite sensitive to the load conditions [4]: to facilitate the 3D scanning, the two ends of the real tape sample are bonded together transversely, while for FE shape, the whole model is constrained longitudinally.

In Fig. 10-c, the slight undulations in the FE shape are due to the relatively large element size compared to the resolution of the laser-scanning system. The transverse curvature is calculated through the scanned region, Fig. 10-d, and it agrees well with the FE shape. Therefore, the parametric study is focused on the ploy region B.

\section{E. Parametric study}

A parametric study on the governing factors of the folded tape shape is performed using the FE model. Fig. 11-a shows the ploy length $L^{*}$ (Eq.9) as a function of sample width, $b$, which is dominated by subtended angle: the initial radius and thickness are kept as $12.5 \mathrm{~mm}$ and $0.65 \mathrm{~mm}$. With an increase in sample width, i.e. subtended angle, the ploy region $\mathrm{B}$ also increases. The ploy length data points from the FE model are determined with different accuracies. $\chi_{y}$ at point $\mathrm{P}$ (Fig. 9) is $\sim 10 \% \cdot 1 / R$ for $b=25 \mathrm{~mm}\left(120^{\circ}\right.$ in subtended angle), $\sim 5 \% \cdot 1 / R$ with $b=40 \mathrm{~mm}\left(180^{\circ}\right.$ in subtended angle), and $<1 \% \cdot 1 / R$ for $b \geq 50 \mathrm{~mm}$. For the tapes with a constant subtended angle of $180^{\circ}$, the folding behaviour with same $L / b$ ratio is expected to be similar, except their loading magnitudes. Fig. 11-b shows the effects of initial radius, $R$, on the $L^{*}$. The theoretical predictions of the $L^{*}$ closely match the FE model: data were extracted with $\chi_{y}$ lying between $2 \%-5 \%$ of $1 / R$ in each case at point $\mathrm{P}$. By increasing the sample thickness, Fig. 11$\mathrm{c}$, the ploy length decreases. The analytical model for $L^{*}$ is developed based on thin-shell theory (with typically $t / R<$ $1 / 20$ [26]), its effectiveness reduces with increasing in tape thickness: $\chi_{y}<10 \% \cdot 1 / R$ is adopted for $t \leq 1 \mathrm{~mm}$, and $\chi_{y}$ is $20 \%$ of the original curvature with $t=2.6 \mathrm{~mm}$.
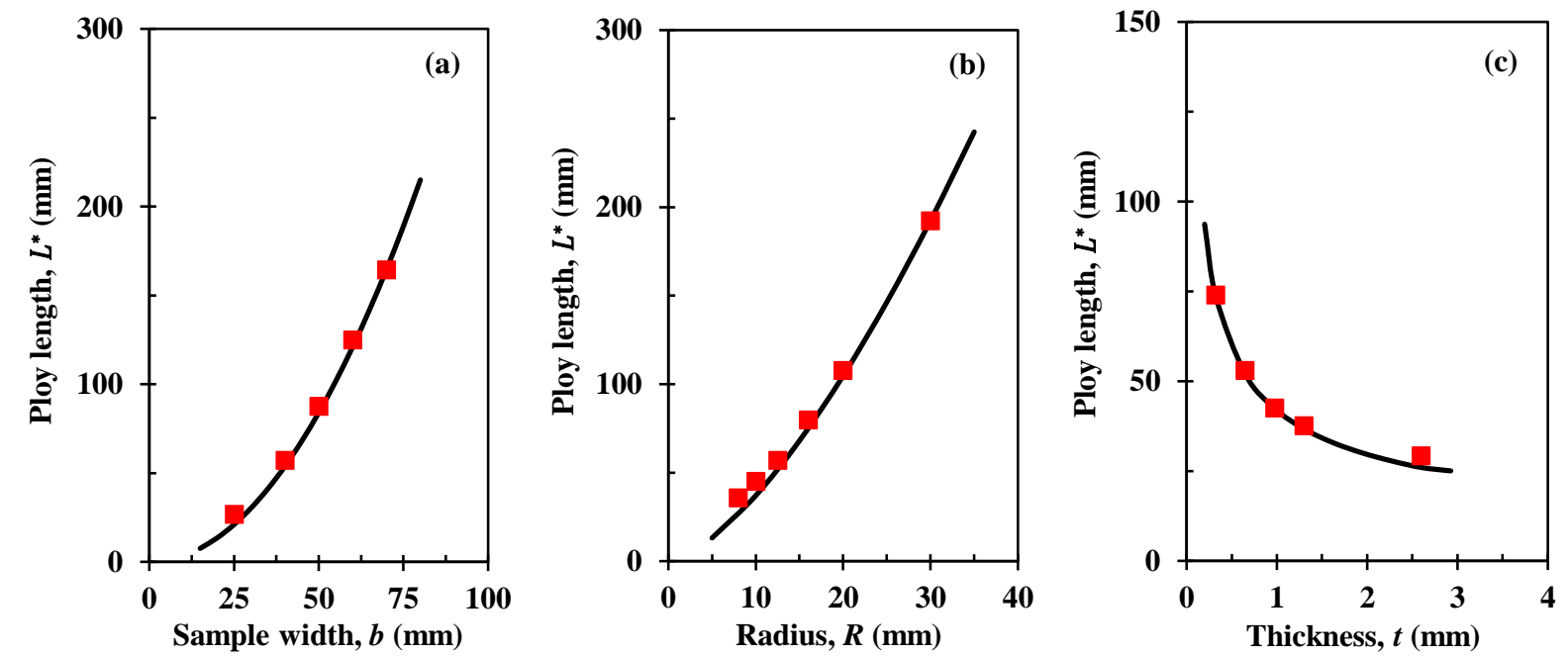

Fig. 11 Effects of (a) sample width, (b) initial radius and (c) thickness on ploy length $L^{*}$. Solid lines are analytical predictions using Eq. 9; data points are extracted from the FE model.

\section{Conclusions}

In this research, we evaluated the shape of a composite tape-spring structure. Both the shape-changing process during folding and the static folded shapes are evaluated. The "free" bending set-up with minimal constraints enable the folding to be studied in great details. An FE model is established following the Naik's idealisation, and calibrated by experimental results.

The torsional buckling is clearly demonstrated by curvature changes from the FE model; while experiments show more gradual and smooth response owing to the presence of initial imperfections. The folded tape shape from the FE model is also compared to the real composite tape. Owning to the differences in applied boundary conditions, the central fold region is not comparable, while the ploy region from both methods are effectively the same. The 
transverse curvature throughout the ploy shape follows the exponential decay. Our analytical model provides a good prediction of the folded tape shapes.

\section{Acknowledgments}

Financial support from Innovate UK (Grant No. 113077, RG82506) and the lead partner Safran Landing Systems

Ltd., UK, are acknowledged. Technical supports from the Materials and Structures Laboratory, Department of Engineering are also acknowledged.

\section{References}

[1] Lachenal X, Daynes S, Weaver PM. Review of morphing concepts and materials for wind turbine blade applications. Wind Energy 2013;16:283-307.

doi: $10.1002 /$ we. 531

[2] Rimrott FPJ. Storable tubular extendible member: a unique machine element. Machine Design 1965;37:156-165.

[3] Schultz MR, Hulse MJ, Keller PN, Turse D. Neutrally stable behavior in fiber-reinforced composite tape springs. Composites Part A 2008;39:1012-1017.

doi: 10.1016/j.compositesa.2008.03.004.

[4] Seffen KA. On the behavior of folded tape-springs. Journal of Applied Mechanics 2001;68:369-375. doi: 10.1115/1.1365153

[5] Yee JCH, Pellegrino S. Folding of woven composite structures. Composites Part A 2005;36:273-278. doi: 10.1016/j.compositesa.2004.06.017

[6] Rimrott FPJ. Two secondary effects in bending of slit thin-walled tubes. Journal of Applied Mechanics 1966;33:75-78. doi: $10.1115 / 1.3625028$

[7] Calladine CR. Theory of shell structures. Cambridge: Cambridge University Press; 1988. doi: $10.1017 / \mathrm{CBO} 9780511624278$

[8] Mansfield EH. The bending and stretching of plates. 2nd ed. Cambridge: Cambridge University Press; 1989. doi: 10.1017/CBO9780511525193

[9] Seffen KA. Analysis of structures deployed by tape-springs. PhD Thesis. University of Cambridge, 1997.

[10] Schmidt RK. Aircraft landing gear assembly. EP3069991B1, 2017.

[11] Wang B, Seffen KA, Guest SD. Folding of a bistable tape-spring structure based on plain-woven composite. Proceedings of the $26^{\text {th }}$ International Conference on Coposites/Nano Engineering, Paris: 2018.

[12] Seffen KA, Wang B, Guest SD. Folded orthotropic tape-springs. Journal of the Mechanics and Physics of Solids 2018. doi: 10.1016/j.jmps.2018.09.017

[13] Naik NK, Ganesh VK. An analytical method for plain weave fabric composites. Composites 1995;26:281-289. doi:10.1016/0010-4361(95)93671-6

[14] MatWeb Materials Information Database. E-glass fiber, general. http://www.matweb.com (accessed Nov 13, 2017).

[15] MatWeb Materials Information Database. Overview of polypropylene. http://www.matweb.com (accessed Nov 15, 2017).

[16] EOS Technologies Inc. zSnapper manual (v3.2). 2011.

[17] The MathWorks Inc. MATLAB Release R2017b. Natick, Massachusetts, United States: 2017.

[18] Ansar M, Xinwei W, Chouwei Z. Modeling strategies of 3D woven composites: A review. Composite Structures 2011;93:1947-1963. doi: 10.1016/j.compstruct.2011.03.010

[19] Akkerman R. Laminate mechanics for balanced woven fabrics. Composites Part B 2006;37:108-116. doi: 10.1016/j.compositesb.2005.08.004

[20] Mallol P, Mao H, Tibert G. Experiments and simulations of the deployment of a bistable composite boom. Journal of Spacecraft and Rockets 2017:1-11. doi: 10.2514/1.A33906

[21] Hill R. Elastic properties of reinforced solids: Some theoretical principles. Journal of the Mechanics and Physics of Solids 1963;11:357-372. doi: 10.1016/0022-5096(63)90036-X

[22] Hashin Z, Shtrikman S. A variational approach to the theory of the elastic behaviour of multiphase materials. Journal of the Mechanics and Physics of Solids 1963;11:127-140. doi: 10.1016/0022-5096(63)90060-7

[23] Abaqus User Manual. Abaqus theory guide. Version 6.14. Dassault Syst Simulia Corp 2014.

[24] Schindelin J, Arganda-Carreras I, Frise E, Kaynig V, Longair M, Pietzsch T, et al. Fiji: an open-source platform for biologicalimage analysis. Nature Methods 2012;9:676-682. doi: 10.1038/nmeth.2019

[25] Sherburn M, Brown L. TexGen open source project V3.10.0 2017. http://texgen.sourceforge.net/.

[26] Qatu MS, Sullivan RW, Wang W. Recent research advances on the dynamic analysis of composite shells: $2000-2009$. Compos Struct 2010;93:14-31. doi: 10.1016/j.compstruct.2010.05.014 